\title{
Comparative Analysis of MC-CDMA System with Wavelet Packet Based MC-CDMA System Using Different Modulation Techniques
}

\author{
Manmohan Singh Chandoliya ${ }^{1}$, Rajni Jainwal ${ }^{2,}$ Om Prakash Mahela ${ }^{3}$ \\ ${ }^{I}$ (M.Tech. Scholar Yagyavalkya Institute of Technology, Jaipur, India) \\ ${ }^{2}$ (M.Tech. Scholar, Sanghvi Institute of Technology, Indore, India) \\ ${ }_{3}^{3}$ (PhD. Scholar, Indian Institute of Technology, Jodhpur, India)
}

\begin{abstract}
Recently conventional Multi-Carrier Code Division Multiple Access (MC-CDMA) techniques attract more attention of researcher's due to its high frequency spectrum efficiency and high data rate transmission. MC-CDMA is a Multiple access scheme used in Orthogonal Frequency Division Multiplexing (OFDM) based telecommunication systems, allowing the system to support multiple users at the same time. Specialized wavelet packet waveform set is used as the modulation waveform in a multicarrier CDMA system. The wavelet packet waveform is the waveform generated from a full binary wavelet packet tree. This paper presents a design of conventional MC CDMA and wavelet packets based MC CDMA and their comparison on the basis of performance matrices. The performance parameter used in this comparison is Bit Error Rate (BER). The spectral analysis is done by wavelet.
\end{abstract}

Keywords: OFDM, MC-CDMA, WPMC-CDMA, AWGN Channel, Bit Error Rate.

\section{Introduction}

Multi carrier CDMA technique has become popular in wireless communications, mainly due to its high spectral frequency, frequency selective fading and flexibility to support integrated applications [1]. MC-CDMA is the combination and improvement of CDMA technique and OFDM parallel transmit technique [2]. In [3], authors presented the concept of combined equalization for uplink MC-CDMA and perform a theoretical analysis which shows that better single-user bounds than the classical matched-filter bounds are achieved with the proposed concept. In [4], authors proposed a novel fractionally spread multicarrier CDMA arrangement i.e. the FS MC-CDMA scheme, which employs both T-domain spreading and F-domain spreading. Specifically the employment of concatenated T-domain spreading for improving the achievable performance, when communicating over wireless channels exhibiting both frequency-selective and time-selective fading is presented. In [5], authors proposed a multicarrier direct sequence code division multiple access, the so called pre-multi-coded MC-DS-CDMA system. In this scheme, the basic MC-DS-CDMA system is augmented with a pre-coder and a multi-code encoder at the transmitter. A frequency based multiple access architecture, frequency division multi carrier CDMA (FD-MC-CDMA) is proposed in [6]. The proposed architecture combines the best elements of FDMA and MC-CDMA to simultaneously exploit frequency diversity and minimize multiple access interface (MAI). In [7], authors proposed a technique for interference suppression in multicarrier code-division access systems which exploit the structural differences in signals that arrive at the receiver with Doppler shifts or carrier offsets. In [8], authors presented a new method of using MC-CDMA as transmission scheme, instead of Orthogonal Frequency Division Multiplexing in WiMAX based on the IEEE 802.16 standards.

In this paper, a specialized wavelet packet waveform set, i.e., the waveform generated from a full binary wavelet packet tree, is used as the modulation waveform in a multicarrier CDMA system. A novel receiver is designed that utilizes the time domain localization property of the wavelet packets. In this design multipath signals within one chip period are combined in the time domain to achieve time-domain diversity in a manner similar to the conventional RAKE receiver design. Each RAKE finger uses a wavelet packet transform to demodulate the corresponding path of the multicarrier signal in the time-domain rather than the frequency domain. The demodulated signal is then de-spread using the corresponding spreading code [9]. Compared with WP Based MC-CDMA and conventional MC-CDMA, the need of guard intervals in WP MC CDMA or MCCDMA is eliminated by using WP time diversity modulation (FMT) in wireless application [10]. The spectra of each sub carrier in WP approach are overlapped, resulting in more efficient use of the spectrum. In other words, the orthogonally of the transmitted waveforms is achieved not by either cyclic prefix or non-overlapping sub channels, but rather by making use of the unique simultaneous time and frequency localization properties of the WP which are not achievable by the conventional MC-CDMA and WP MC-CDMA. This is similar in spirit to the pulse-shaped conventional MC CDMA. The complete set of wavelet waveforms are used instead of only one wavelet. Waveform helps to exploit explicitly the introduced time-diversity in a RAKE receiver design. 
This paper presents performance analysis of wavelet packet based MC CDMA in comparison with the conventional MC-CDMA using Additive White Gaussian Noise (AWGN) Channel in term of bit error rate (BER). This paper is organized as follows: Section II describes the system modeling. Section III describes the Bit Error Rate and finally the simulation results and their discussions are presented in section IV. Finally the section $\mathrm{V}$ includes the concluding remark.

\section{System Modeling}

The CDMA system consists of transmitter as well as receiver. The details of MC-CDMA receiver/transmitter and WP MC-CDMA receiver/transmitter are given in the sections following.

\subsection{MC-CDMA Transmitter}

An OFDM carrier signal is the sum of number of orthogonal sub-carriers, with baseband data on each sub-carrier being independently modulated commonly using some type of quadrature amplitude modulation (QAM) or phase-shift keying (PSK). This composite baseband signal is typically used to modulate a main RF carrier. S (n) is a serial stream of binary digits. By inverse multiplexing, these are first de multiplexed into $\mathrm{N}$ parallel streams, and each one mapped to a (possibly complex) symbol stream using some modulation constellation (QAM, PSK, etc.). An inverse FFT is computed on each set of symbols, giving a set of complex time-domain samples. These samples are then quadrature-mixed to pass band in the standard way [11]. The transmitter of MC-CDMA is shown in Fig. 1.

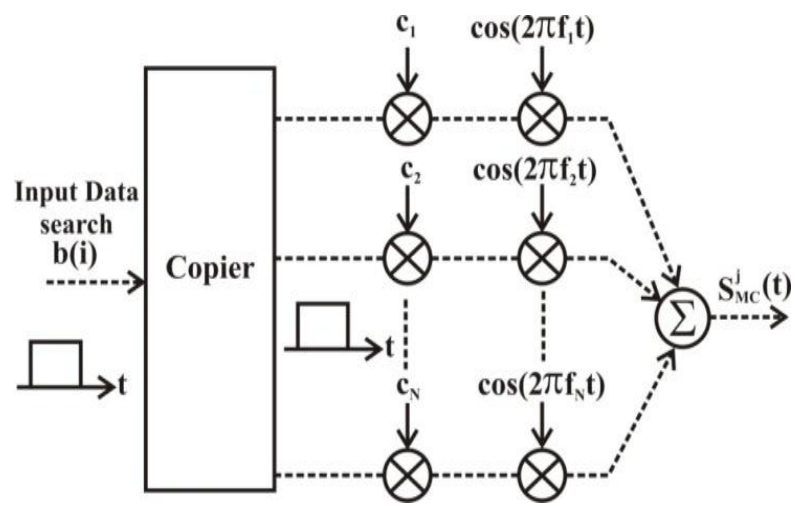

Fig. 1. Transmitter of MC-CDMA

\subsection{MC-CDMA Receiver}

The receiver picks up the signal $r(t)$, which is then quadrature-mixed down to baseband using cosine and sine waves at the carrier frequency. This also creates signals centered on $2 \mathrm{fc}$, so low-pass filters are used to reject these. The baseband signals are sampled and digitized using analog-to-digital converters (ADCs), and a forward FFT is used to convert back to the frequency domain. This returns $\mathrm{N}$ parallel streams, each of which is converted to a binary stream using an appropriate symbol detector. These streams are then re-combined into a serial stream, $\mathrm{s}(\mathrm{n})$ which is an estimate of the original binary stream at the transmitter [12]. The receiver of MCCDMA is shown in Fig. 2.

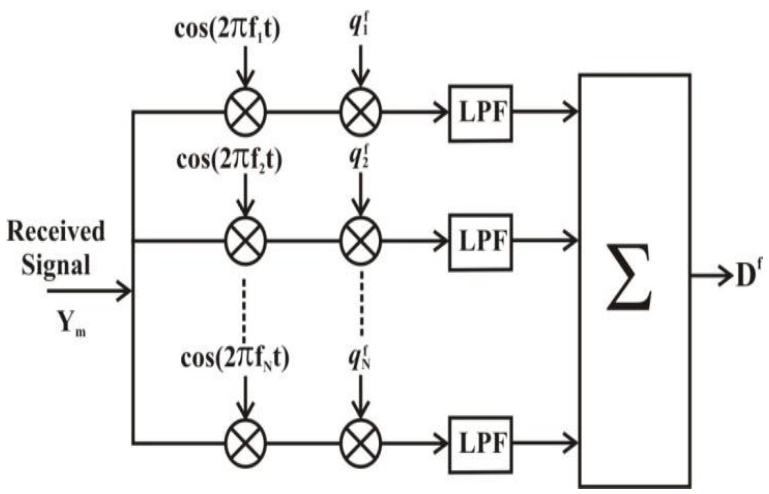

Fig. 2. Receiver of MC-CDMA 


\subsection{Transmitter of WP MC-CDMA}

Although a number of different schemes are proposed in the literature, the multicarrier CDMA schemes can be categorized mainly into two groups.

- $\quad$ First one spreads the original data stream using a given spreading code, and then modulates a different sub carrier with each chip (the spreading operation in the frequency domain).

- $\quad$ Second spreads the serial-to-parallel (S/P) converted data streams using a given spreading code, and then modulates a different sub carrier with each of the data stream ( the spreading operation in the time domain). One group spreads the user symbols in the frequency domain and the other spread user symbols in the time domain. Wavelet Packets have the property of both time and frequency localization. The transmitter of WP-MCCDMA is shown in Fig. 3.

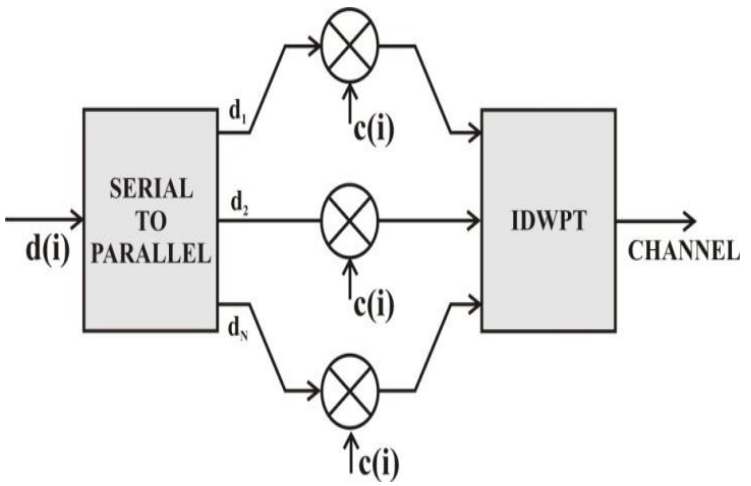

Fig. 3. Transmitter of WP-MC-CDMA

\subsection{Receiver of WP MC-CDMA}

A series of delayed version of the received signals are detected by single path detectors. In Each single path detector, a DWPT (Digital Wavelet Packet Transform) block is used for demodulation of the signal for the corresponding resolved path. The multi-user interference can be effectively eliminated if the desired user spreading code is known, which is assumed true in the following. The DWPT demodulated signal is forwarded to the dispreading part to obtain a detected decision variable for the resolved path [13]. The receiver of WP-MCCDMA is shown in Fig. 4.

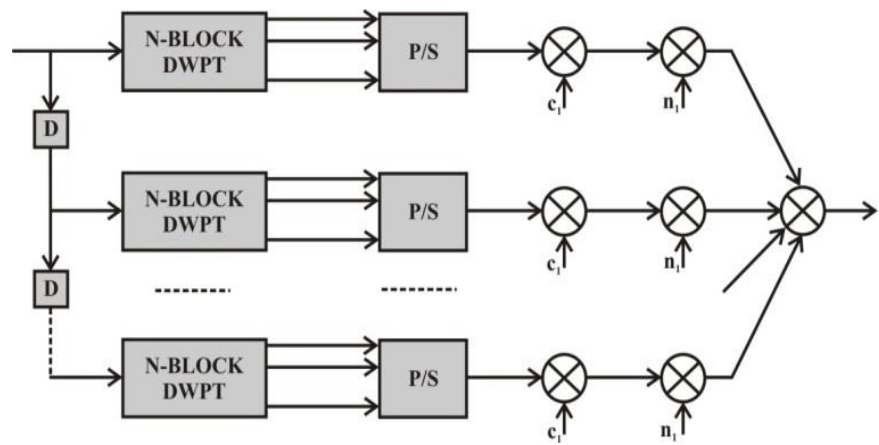

Fig. 4. Receiver of WP MC CDMA

\section{Bit Error Rate}

The bit error rate or bit error ratio (BER) is the number of bit errors divided by the total number of transferred bits during a studied time interval. The bit error rate of BPSK in AWGN can be calculated as BER= Error/ total number of bit [14].

\section{Simulation Results and Discussion}

In this section, we have presented various BER vs. SNR Plots. Performance of the MC-CDMA and wavelet packet based MC-CDMA is shown in Fig. 5 and Fig. 6 respectively for AWN Channel. Simulation results in Fig. 5 and Fig. 6 show that the advantage of BPSK modulation technique for the conventional MCCDMA and wavelet packet based MC-CDMA and wavelet packet based MC-CDMA system BPSK modulation is quite satisfactory as compared to other modulation techniques in AWGN channel. Performance of one user MC-CDMA for AWGN is carried out using BPSK (Binary phase shift key) and QPSK (Quadrature phase shift key) following modulation techniques. 


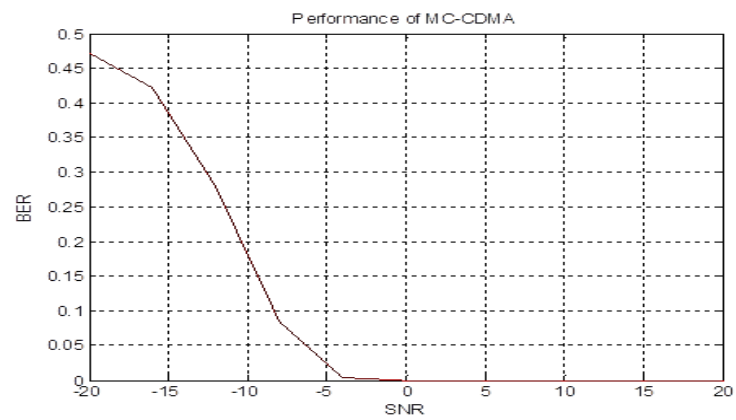

Fig. 5. Performance analysis of BER using BPSK Technique

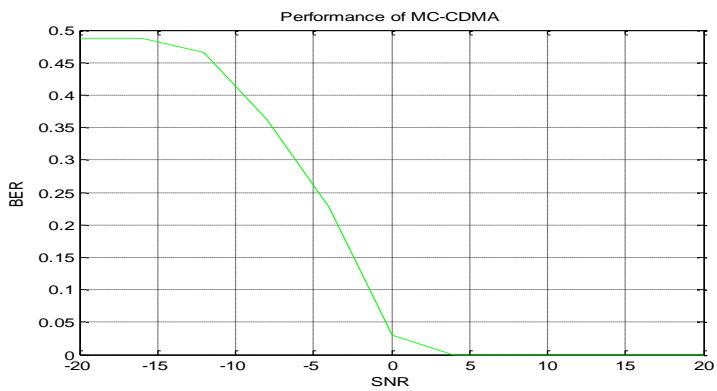

Fig. 6. Performance analysis of BER using QPSK Technique

Performance of WP MC-CDMA for two users in AWGN channel is shown in Fig. 7 and performance of MC-CDMA for two users in AWGN channel is shown in Fig. 8.

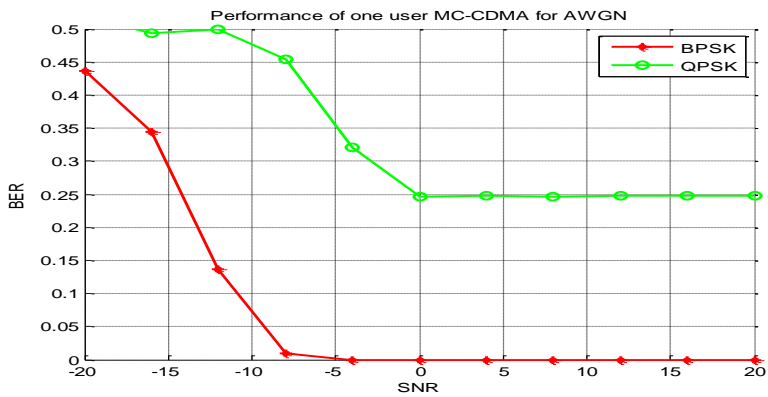

Fig. 7. WP MC CDMA System for 2 users

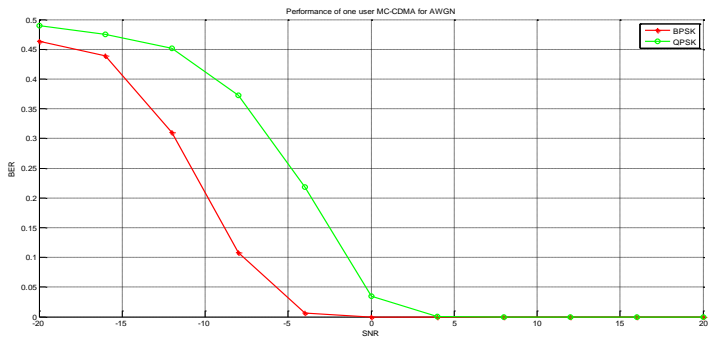

Fig. 8. MC CDMA System for 2 users

\section{Conclusion}

In this paper, comparative analysis of MC-CDMA system with wavelet packet based MC-CDMA system using different modulation techniques is presented. The analysis is carried out for one user as well as two users. The results presented show the performance of different modulation techniques. Wavelet packet based MC-CDMA system BPSK modulation is quite satisfactory as compared to other modulation techniques in AWGN channel. 


\section{References}

[1]. Rui Fa, and Pei Xiao, "Joint data detection and phase recovery for downlink MC-2D-CDMA systems," IEEE Transaction on communications, Vol. 57, No. 9, pp. 2782-2789, September 2009.

[2]. Xiangbin Yu, and Guangguo Bi, "Performance of complex orthogonal wavelet packet based MC-CDMA system with space-time coding over rayleigh fading channel," in Proceeding IITA International Conference on Control, Automation and Systems Engineering," Zhangjiajie, July 2009, pp. 136-139.

[3]. Ivan Cosvic, Michael Schnell, and Andreas Springer, "Combined equalization for uplink MC-CDMA in rayleigh fading channels," IEEE transactions on Communications, Vol. 53, No, 10, pp. 1609-1614, October 2005.

[4]. Lie-Liang Yang, and Lajos Hanzo, "Performance of fractionally spread multicarrier CDMA in AWGN as well as slow and fast Nakagami-m fading channels," IEEE Transactions on Vehicular Technology, Vol. 54, No. 5, pp. 1817-1827, September 2005.

[5]. Yewen Cao, Tjeng Thiang Tjhung, and Chi Chung Ko, "Performance of a new premulticoded multicarrier DS-CDMA system in Nakagami fading," IEEE Transactions on Communications, Vol. 55, No. 7, pp. 1363-1372, July 2007.

[6]. Zhiqiang Wu, and Carl R. Nassar, "FD-MC-CDMA: A frequency -based multiple access architecture for high performance wireless communication," IEEE Transactions on Vehicular Technology, Vol. 54, No. 4, pp. 1392-1399, July 2005.

[7]. Brandon Hombs, and James S. Lehnert, "Multiple-access interface suppression for MC-CDMA by frequency-domain oversampling," IEEE Transactions on Communications, Vol. 53, No. 4, pp. 677-686, April 2005.

[8]. Rabah W. Aldhaheri, and Ali H. Al-Qahtani, "Performance analysis of fixed and mobile WiMAX MC-CDMA-based system," in Proceeding $7^{\text {th }}$ International Symposium on Wireless Communication Systems," York, September 2010, pp. 436-440.

[9]. Hongbing Zhang, H. Howard Fan, and Alan R. Lindsay, "Receiver design for wavelet-based multicarrier CDMA communications", IEEE Transactions on Vehicular Technology, Vol. 54, No. 2, pp. 615-628, April 2005.

[10]. Sandberg, S. D., and Tzannes M. A., "Overlapped discrete multitone modulation for high speed copper wire communications," IEEE Journal on Selected Areas on Communications, Vol. 13, No. 9, pp. 1571-1585, December 1995.

[11]. Zexian Li, and Mutti Latva-aho, "Error probability of interleaved MC-CDMA systems with MRC receiver and correlated Nakagami-m fading channels," IEEE Transactions on Communications, Vol. 53, No. 6, pp. 919-923, June 2005.

[12]. Paulo Silva, and Rui Dinis, "Joint turbo equalization and multiuser detection of MC-CDMA signals with low envelope fluctuations," IEEE Transactions on Vehicular Technology, Vol. 58, No. 5, pp. 2288-2297, June 2005.

[13]. Xiangbin Yu, Xiangdong Zhang, and Guangguo Bi, "Performance of an MC-CDMA system based on the complex wavelet packet and pre-equalization technique," IEE Proc. Communications, Vol. 151, No. 2, pp. 152-156, 2004.

[14]. Christos Masouros, and Emad Alsusa, "Two-stage transmitter precoding based on data-driven code-hopping and partial zero forcing beamforming for MC-CDMA communications," IEEE Transactions on Wireless Communications, Vol. 8, No. 7, July 2009.

\section{Biographies}

Manmohan Singh Chandoliya was born in Jaipur in the Rajasthan state of India, on October 10, 1991. He

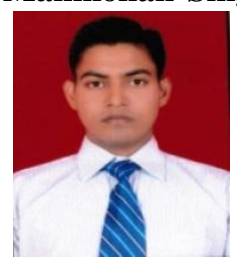
studied at Govt. Engineering College, Bharatpur, India and received the Bachelor of Technology degree in electronics and communication engineering degree from Rajasthan technical University (RTU), Kota, India in 2011. He is currently pursuing M. Tech. in digital communication from RTU, Kota.

Since 2011, he has been lecturer with Indian Institute of Computer Science, Jaipur, India. His special field of interest includes the digital communication, antenna and VLSI technology.

Rajni Jainwal was born in Kota in the Rajasthan state of India, on August 10, 1980. She received the

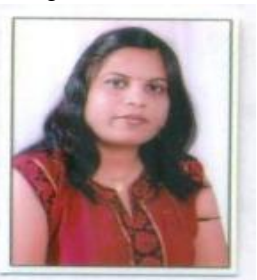
Electronics and Communications engineering degree in 2004. She received M.B.A. from Central India Institute of Management in 2008. She is currently pursuing M. Tech. in digital Communications.

From 2004 to 2005, she was Lecturer with the Satya Sai Institute of Technology and Science, Bhopal, India. From 2005 to 2009, she was Lecturer with Rishi Raj Institute of Technology and Science, Indore, India. Since 2009, she has been Lecturer with S.D. Bansal, Institute of Technology and Science, Indore, India.

Om Prakash Mahela was born in Sabalpura (Kuchaman City) in the Rajasthan state of India, on April 11,

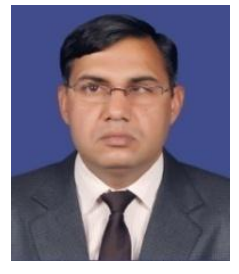
1977. He studied at Govt. College of Engineering and Technology (CTAE), Udaipur, and received the electrical engineering degree from Maharana Pratap University of Agriculture and Technology (MPUAT), Udaipur, India in 2002. He received M.Tech.(Electrical Engineering) with power system specialization in 2013. He is currently pursuing $\mathrm{PhD}$ from Indian Institute of Technology, Jodhpur, India.

From 2002 to 2004, he was Assistant Professor with the RIET, Jaipur. From 2004 to 2013 he has been Junior Engineer-I and presently working as Assistant Engineer with the Rajasthan Rajya Vidhyut Prasaran Nigam Ltd., Jodhpur, India. His special fields of interest are Transmission and Distribution (T\&D) grid operations, Power Electronics in Power System, Power Quality, Load Forecasting and Integration of Renewable Energy with Electric Transmission and Distribution Grid, Applications of AI Techniques in power system. He is an author of 29 International Journals and Conference papers. He is Member of IEEE. He is Member of IEEE Power \& Energy Society. He is Fellow Member of IAEME. He is Reviewer of TJPRC International Journal of Electrical and Electronics Engineering Research. Mr. Mahela is recipient of University Rank certificate from MPUAT, Udaipur, India, in 2002. He is recipient of Gold medal in 2013. 